Cambouis, la revue des sciences sociales aux mains sales

\title{
Accéder au terrain, s'y maintenir, le quitter
}

\section{Du tourment ethnographique au tourment militant en milieu partisan}

Kévin Delasalle

Centre Nantais de Sociologie (CENS, UMR 6025 - Université de Nantes)

kevin.delasalle.univ.nantes@gmail.com

Résumé : S’appuyant sur une thèse de sociologie consacrée au «tourment militant » au sein du Parti Socialiste et fondée sur une enquête par immersion ethnographique, l'article se propose de revenir sur les trois étapes de cette immersion - l'accès au terrain, le maintien sur celui-ci, la sortie du terrain. Seront ainsi interrogées, pour chacune d'entre elles, les stratégies plus ou moins conscientes mises en œuvre par le chercheur pour négocier sa place sur le terrain, passant progressivement du statut de jeune doctorant sympathisant à celui de « militant professionnalisé ». Assumer la double identité de militant et de chercheur nécessite de concilier, d'un côté, l'établissement et l'entretien de relations interpersonnelles sur les différentes scènes du monde militant local avec, de l'autre côté, les logiques d'objectivation qu'impose l'enquête. L'article permet ainsi de soulever la question de la négociation de la présence du chercheur sur son terrain (s'assurer des cooptations, faire ses preuves, donner des gages de fidélité...) et de l'évolution des formes que peut prendre cette négociation au cours d'une recherche par immersion ethnographique de longue durée. En offrant un accès à une intimité militante difficilement explorable par d'autres voies méthodologiques que celle ici analysée, la recherche a finalement rendu possible une sociologie «par le soi » du « tourment militant ».

Date de publication : $30 / 06 / 2021$

Ethnographie, négociation, tourment militant, militantisme, engagement, parti politique, Parti socialiste

Dossier : Pratiques et politiques de la négociation pour accéder et se maintenir sur un terrain d'enquête

Comment citer $: 10.52983 /$ crev.vi0.75

Licence: Cambouis publie ses contenus selon les termes de la Licence Creative Commons Attribution - Pas d'Utilisation Commerciale - Pas de Modification 4.0 International. Les auteurices gardent leurs droits de propriété intellectuelle pleine et entière sur leurs articles. 


\section{Accéder au terrain, s'y maintenir, le quitter}

\section{Du tourment ethnographique au tourment militant en milieu partisan}

\section{Kévin Delasalle}

Centre Nantais de Sociologie (CENS, UMR 6025 - Université de Nantes)

kevin.delasalle.univ.nantes@gmail.com

S'appuyant sur ma thèse (Delasalle, 2017), qui interroge la dimension malheureuse de l'engagement militant - le « tourment militant »-à partir d'une enquête menée par immersion ethnographique pendant six ans, entre 2010 et 2016, au Parti Socialiste (PS), le présent article interroge la question de l'accès, du maintien et de la sortie du terrain en milieu partisan.

Au cours de ma recherche, je suis progressivement passé du statut de jeune doctorant sympathisant à celui de «militant professionnalisé ». Assumer l'identité double de militant et de chercheur n'est toutefois pas chose aisée (Broqua, 2009a). Cela nécessite de concilier, d'un côté, l'établissement et l'entretien de relations interpersonnelles sur les différentes scènes du monde militant local avec, de l'autre côté, les logiques d'objectivation qu'impose l'enquête. Il convient, dès lors, de fournir dès à présent quelques précisions quant à mon rapport à l'objet « socialiste ».

Appartenant à la société qu'il souhaite étudier, le sociologue n'est jamais neutre, son rapport au monde social est nécessairement « engagé » et le regard qu'il porte sur son objet de recherche est le fruit de son histoire personnelle et de la position qu'il occupe dans l'univers social qu'il étudie (Bensa, 2008b). Alors que beaucoup de chercheurs possédant un double statut de militant et de chercheur préfèrent le taire ou rester discrets dans le champ universitaire sur leurs engagements - Rémi Lefebvre rappelle que « sur ce type de questions, une certaine discrétion, mêlée de pudeur, est en général stratégiquement de mise » (Lefebvre, 2010a, p. 128) - j'ai au contraire choisi d'intégrer pleinement la réflexion sur ce sujet à mon travail de thèse.

Avant d'accéder à mon terrain de recherche, celui-ci ne m'était pas entièrement inconnu, du fait de ma socialisation familiale, d'une part ${ }^{1}$, mais aussi pour l'avoir déjà fréquenté, en tant qu'apprenti sociologue, à l'occasion d'une recherche de Master 2 portant sur le rapport du PS aux classes populaires. Je n'étais cependant pas militant socialiste avant de débuter mon immersion ethnographique. À l'image de Flora Bajard, j'étais un « "ethnologue-métis", personne qui étudie un milieu qu'elle connait, mais dont elle ne fait pas partie en raison de ses expériences et appartenances propres » (Bajard, 2013, p. 10).

Néanmoins, la «participation » prenant progressivement, à mesure de l'avancée de ma recherche, le pas sur l'« observation » dans un milieu qui ne m'était pas entièrement étranger, je suis progressivement devenu un « indigène » (Bensa, 2008a) ; jusqu'à finalement endosser trois années durant un costume de militant « professionnalisé », celui de collaborateur d'élus.

Lors de mon immersion, entre 2010 et 2016, des relations se sont par conséquent nouées avec celles et ceux qui sont progressivement devenus des « camarades » : des relations d'amitié parfois, de franche camaraderie souvent, d'inimitié à quelques (rares) occasions. Bien que rompant avec la «neutralité axiologique » attendue dans l'idéal et dans l'idéel, les liens tissés avec ces personnes qui ne sont pas restées de simples « enquêtés » se sont avérés décisifs 
pour l'enquête. Loin de nuire à la recherche, ils m'ont au contraire permis d'accéder à une intimité autrement inaccessible au chercheur. C'est la nature des relations entretenues avec les militants qui, de manière simultanée, a construit l'objet de la recherche tout en permettant son élucidation.

La proximité entretenue avec les militants m'a ainsi offert un accès unique à l'expression de leurs « tourments » qui, autrement, seraient sans doute restés impénétrables. Plus que cela, c'est en partageant leur désarroi, leur malaise, en éprouvant moi-même intimement des émotions identiques aux leurs, qu'il m'a été donné de les comprendre. Par-delà l'imperfection des matériaux ainsi recueillis, il est nécessaire de les réinterroger à la lumière de la trajectoire personnelle du chercheur au cours même de la recherche. Autrement dit, il s'agit là d'assumer pleinement une démarche ethnographique relevant de « l'empirisme irréductible » prôné par Olivier Schwartz et exigeant de « placer la situation d'enquête et ses effets au centre de l'analyse des matériaux ». Il faut dès lors « identifier les rôles » (Schwartz, 1993, p. 274) occupés par le chercheur et interroger leur évolution au fur et à mesure de l'enquête ainsi que l'effet qu'elle produit sur les matériaux recueillis (Favret-Saada, 1977).

\section{L'évolution des matériaux récoltés}

Lévolution des matériaux récoltés. La nature et la quantité des matériaux recueillis au cours de mon « errance » (Caratini, 2017, p. 128) ethnographique ont variées avec l'évolution de mon positionnement personnel. Lors de la première phase de mon immersion (février 2010 à août 2012), mon statut de militant n'ayant pas (encore) pris le dessus sur celui de chercheur, j'ai recueilli un maximum d'informations sur mon « terrain » et mes « enquêtés», tout en m'efforçant de tenir à jour un « journal de terrain ». Rapidement cependant, à mesure que des responsabilités m'étaient confiées et que j'accédais à des instances fermées du parti, grandissait la sensation de «trahir » ceux qui sont progressivement devenus des « camarades ». Il m'est ainsi devenu de plus en plus difficile de me livrer à l'exercice - chronophage et exigeant - du recueil de données ethnographiques. Lorsque je suis devenu collaborateur d'élus (septembre 2012), mon statut de « chercheur » s'est complétement effacé derrière celui de «militant professionnalisé ». Les matériaux ethnographiques recueillis au cours des trois années suivantes sont par conséquent lacunaires. Mes responsabilités professionnelles et partisanes m'ont cependant permis d'accumuler des matériaux divers et variés qui se sont révélés précieux par la suite (comptes rendus « officiels » de réunions; courriers et mails de militants ; notes militantes personnelles ; articles de presse...). Une fois mon contrat de travail terminé (au cours de l'année 2015), j'ai poursuivi mes activités militantes durant quelques mois, tout en débutant l'écriture de ma thèse. Rompre définitivement avec un milieu militant qui avait occupé tant de place dans ma vie professionnelle et personnelle au cours des années précédentes était « coûteux ». Mon désengagement fut par conséquent progressif. Ce n'est finalement qu'une fois ce processus de « désengagement » en cours d'achèvement que je me suis autorisé à dévoiler à certains de mes « camarades » les plus proches la reprise de mes activités de recherche ainsi que le sujet définitif sur lequel portait ma thèse. Ce n'est qu'à compter de ce moment-là qu'il m'a été possible (et que je me suis autorisé) à effectuer des entretiens formels et approfondis avec huit militants et deux anciens collègues 
collaborateurs d'élus (août 2016 à décembre 2016). Ces entretiens ont porté sur leurs trajectoires (militante, personnelle, professionnelle) ainsi que sur les événements marquants, locaux et nationaux, s'étant déroulés dans la vie partisane entre 2010 et 2016.

L'article entend par conséquent soulever la question de la négociation de la présence du chercheur au sein d'un parti politique (Bachelot, 2011) s'assurer des cooptations, faire ses preuves, donner des gages de fidélité... - et de l'évolution des formes que peut prendre cette négociation, dont l'objectif est de « chercher une voie d'accès adéquate pour tenter de pénétrer dans la maison [...] puis d'évoluer avec le maximum de liberté dans chacune de ses pièces $[\ldots]$ le plus longtemps possible sans être raccompagné promptement à la sortie » (Aït-Aoudia, 2010, p. 15).

Ma recherche s'est déroulée en trois temps, au cours desquels je suis donc progressivement passé du statut de jeune doctorant sympathisant à celui de «militant professionnalisé ». Dans les pages qui suivent, je reviendrai sur ces trois étapes - négocier l'accès au terrain, négocier le maintien sur le terrain, négocier (avec soi-même) la sortie du terrain - en détaillant, pour chacune d'elles, les stratégies, plus ou moins conscientes, mises en place dans la négociation avec les « enquêtés ».

\section{Négocier l'accès au terrain : la progressivité de l'immersion ethnographique en milieu partisan}

S'intéresser à la question de la négociation nécessite de pénétrer dans la « cuisine » du chercheur, souvent passée sous silence (Naudier, Simonet, 2011). Généralement tues ou reléguées dans les notes de bas de page (Fassin, 2008), les « composantes de la relation d'enquête » (Bizeul, 1998, p. 754) peuvent pourtant se révéler particulièrement heuristiques lorsqu'elles sont pleinement intégrées à l'analyse.

\section{Une négociation implicite et à l'usure}

C'est dans l'optique d'étudier l'évolution de l'implantation du PS dans une région de l'Ouest de la France, en suivant notamment les préconisations de Frédéric Sawicki (1988 ; 1997), que débute, au cours de l'année 2010, mon enquête ethnographique. Je n'ai alors qu'une perception relativement floue du devenir de ma recherche, et le « microscope » (Sawicki, 2000) semble être un bon outil pour éclaircir son horizon.

En janvier 2010, deux ans après avoir mené une observation (non-participante) de la campagne des élections municipales et cantonales de 2008 dans le cadre de mon Master 2, je renoue contact avec une élue suivie lors de cette première recherche ${ }^{2}$. Celle-ci m'autorise à suivre la campagne des élections régionales, tout en m'invitant à laisser mon carnet de notes de côté (« En tant que participant cette fois-ci !»). On le comprend : dès le départ, la poursuite d'une observation non-participante allait m'être difficile.

Lors des premières semaines de mon travail de terrain, je ressens en effet une certaine méfiance de la part d'« enquêtés » s'interrogeant sur les raisons de ma présence sur tous les « terrains ». Ayant commencé mon travail d'observation de la vie politique locale en janvier 2009, je suis alors présent lors de nombreux événements (cérémonies de vœux du Maire, séances du Conseil municipal, du Conseil général et du Conseil régional, manifestations...). Ma
2 L'élue en question est également une amie de mes parents ; cela a, bien entendu, facilité mon accès au terrain. 
présence, qui s'y veut incognito, est bien entendu remarquée. Lors de l'un des premiers «boitages » auquel je prends part lors de la campagne des élections régionales, Édith, Conseillère municipale, me fait par exemple remarquer : Édith : « T'étais au Conseil Municipal vendredi ? Je t'ai aperçu sur ton banc. » [Journal de terrain -3 février 2010]

Au cours de cette même période, une assistante du Maire se montre particulièrement curieuse de connaître les raisons de ma présence lors de chacune des cérémonies de vœux rythmant le mois de janvier. Prenant régulièrement quelques photos, tentant de laisser traîner mes oreilles lors de diverses conversations, mon attitude ne peut en effet qu'interroger les collaborateurs du Maire. Je justifie alors ma présence en répondant que j'effectue une thèse sur l'implantation locale du PS, d'où mon intérêt pour ces différents événements auxquels j'assiste avec assiduité. C'est donc non sans surprise que l'assistante en question me voit, un matin du mois de mars 2010, distribuer des tracts sur un marché à l'occasion de la campagne des élections régionales : L'assistante (étonnée) : «Alors tu fais campagne?»

Moi : «Bah oui !»

L'assistante : «Avec ton regard extérieur. »

Moi : «Ouais (Rire).»

Elle me présente à la personne qui l'accompagne : « Il fait la campagne et il fait une thèse sur le Parti Socialiste. C'est ça hein ?»

Moi : «Ouais c'est ça. Je fais campagne (Rire). »

L'assistante : «C'est bien. C'est pour quand ? T'en es où ? »

Moi : «J'en suis où ? J'en suis pour l'instant que... je fais campagne (Rire). »

[Journal de terrain - 6 mars 2010]

Toute l'ambivalence de mon positionnement transparaît dans cet échange. Tentant de faire oublier mon statut de chercheur, je mets en avant celui de militant et reste évasif sur l'avancée de ma recherche.

Dès lors, l'accès au terrain s'apparente à une négociation à l'usure : il s'agit de faire oublier mon statut de chercheur à des enquêtés qui se montrent méfiants sur les raisons de ma présence ${ }^{3}$. Je fais par conséquent preuve d'un activisme militant ostensiblement affiché. Dans une sorte de négociation implicite avec mes enquêtés, il me faut, à ce moment-là de l'immersion, leur donner des gages de loyauté afin d'accéder à mon terrain de recherche. En m'intégrant totalement à la campagne, en devenant moi-même un militant, je dois leur prouver qu'ils peuvent me faire confiance.

Christophe Broqua souligne la difficulté à maintenir un rôle de simple observateur dans une organisation partisane : «L'ethnographe se trouve souvent lui-même surveillé, ce qui renforce son inconfort, et parfois son désir d'offrir une contrepartie à sa présence, qui peut prendre la forme d'un engagement accru » (Broqua, 2009b, p. 382). Il m'était ainsi impossible de simplement «jouer un rôle ». L'ambiguïté de mon statut - ni simple observateur, ni réellement militant - était difficilement tenable sur la longue durée. Pour être intégré, il me fallait devenir militant, en incorporer, à l'occasion de la campagne électorale, les pratiques, savoir-faire et savoir-être.

En donnant l'occasion aux militants de se retrouver presque quotidiennement, en permettant aux nouveaux militants de côtoyer des militants plus expérimentés et des élus, les campagnes électorales participent en effet à l'acquisition par les nouveaux entrants des savoir-faire militants. Véritable « rite intégrateur » (Lefebvre, 2010b, p. 242), période de socialisation intense au militantisme (socialisation «par le corps », apprentissage des pratiques militantes tels que collages, porte-à-porte, tractages..., pratiques de sociabilité et de convivialité dont les pots après les actions militantes, les blagues...), la campagne m'aura ainsi permis de gagner la confiance de mes enquêtés... mais également, conséquence inattendue de l'immersion ethnographique, de prendre goût aux activités militantes.
3 Je (ré)intègre par ailleurs alors un milieu d'interconnaissances dont les frontières avec mon « univers social » sont relativement poreuses (par exemple, certains des étudiants suivant mes cours - je suis Moniteur - sont militants socialistes). 


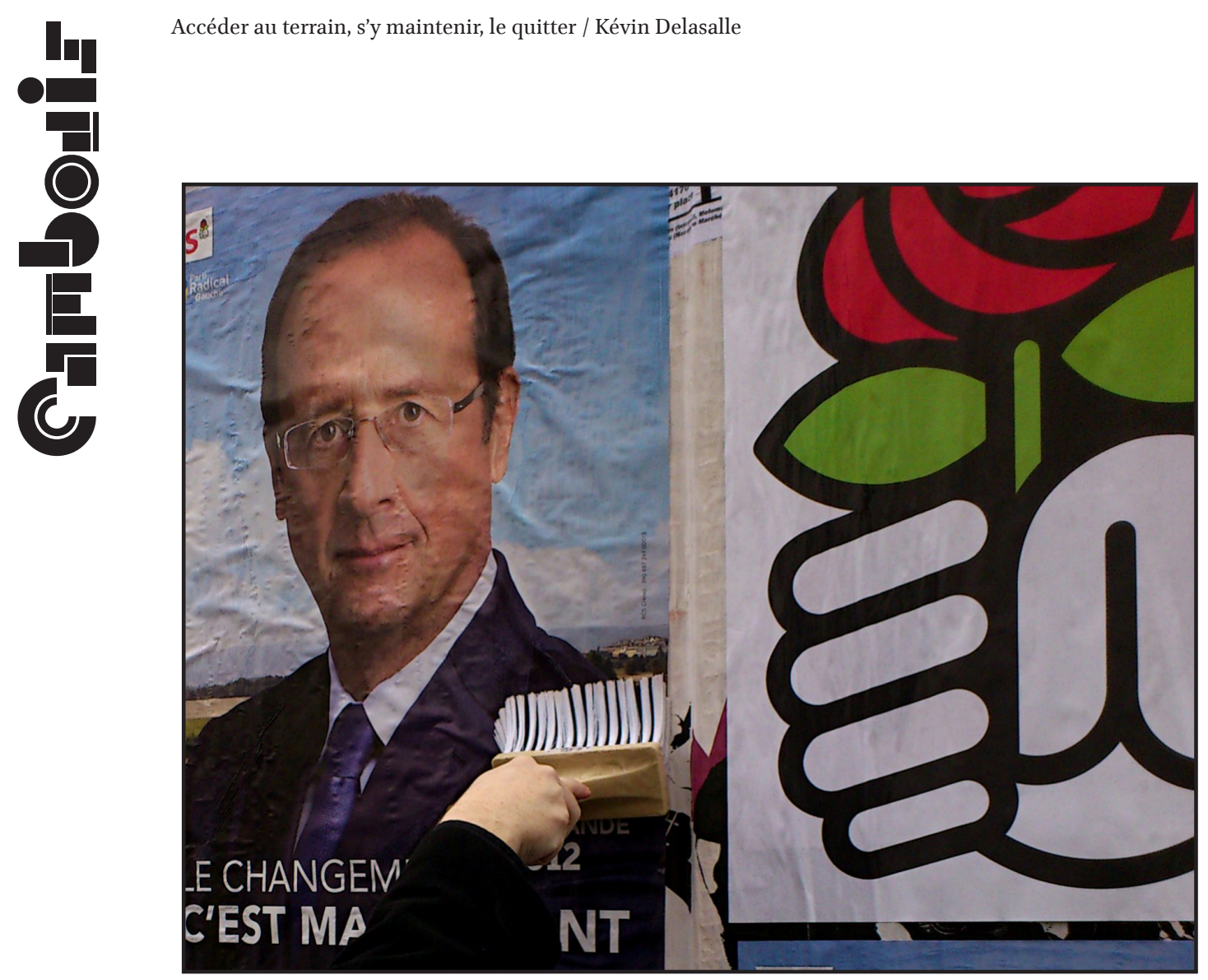

Campagne des élections présidentielles 2012 : quand le militant prend le pas sur l'ethnographe. Collage en faveur de François Hollande [Document personnel de l'auteur]

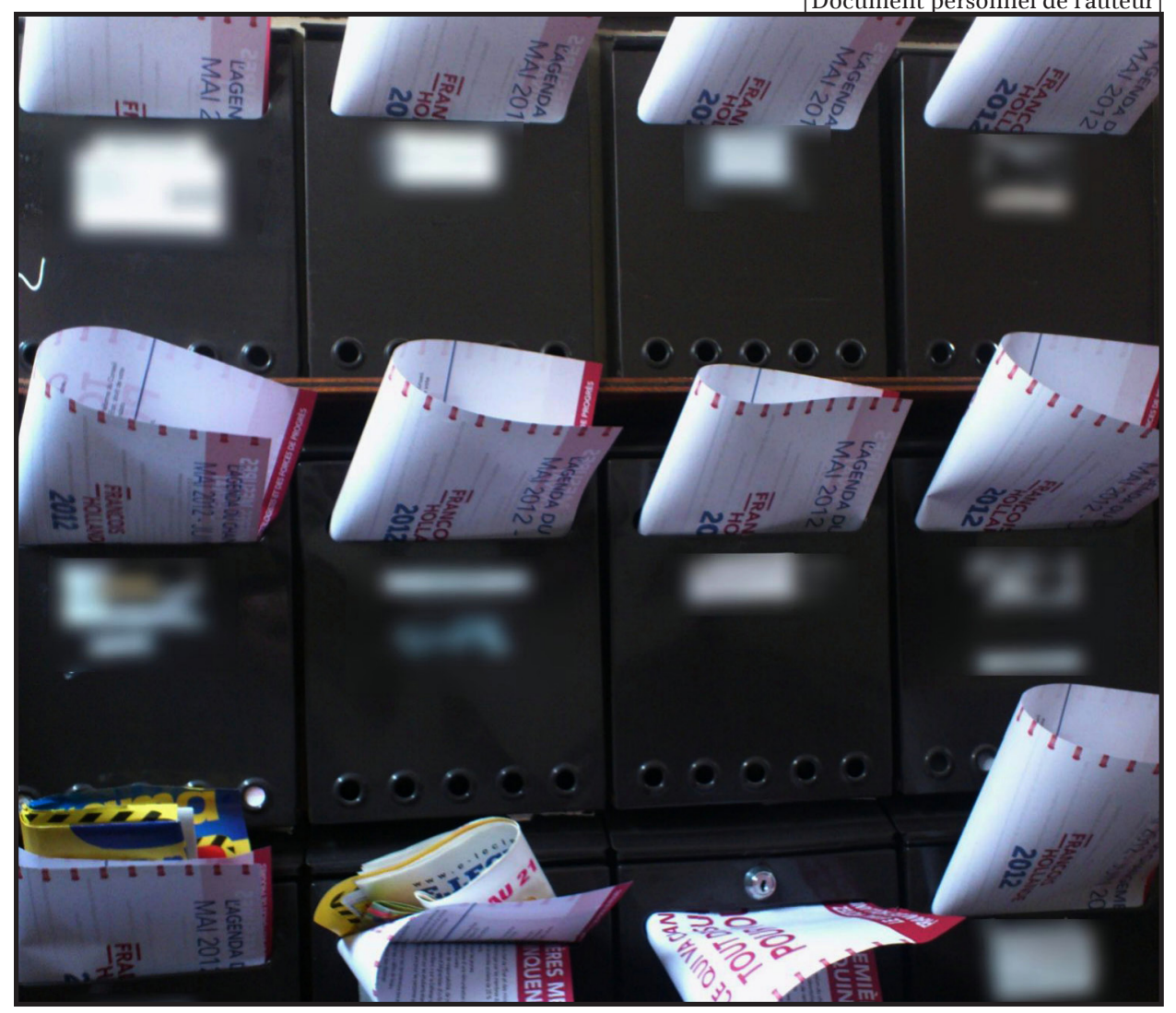

«Boitage » du document présentant « l'agenda du changement » et les « premières mesures du quinquennat» [Document personnel de l'auteur] 


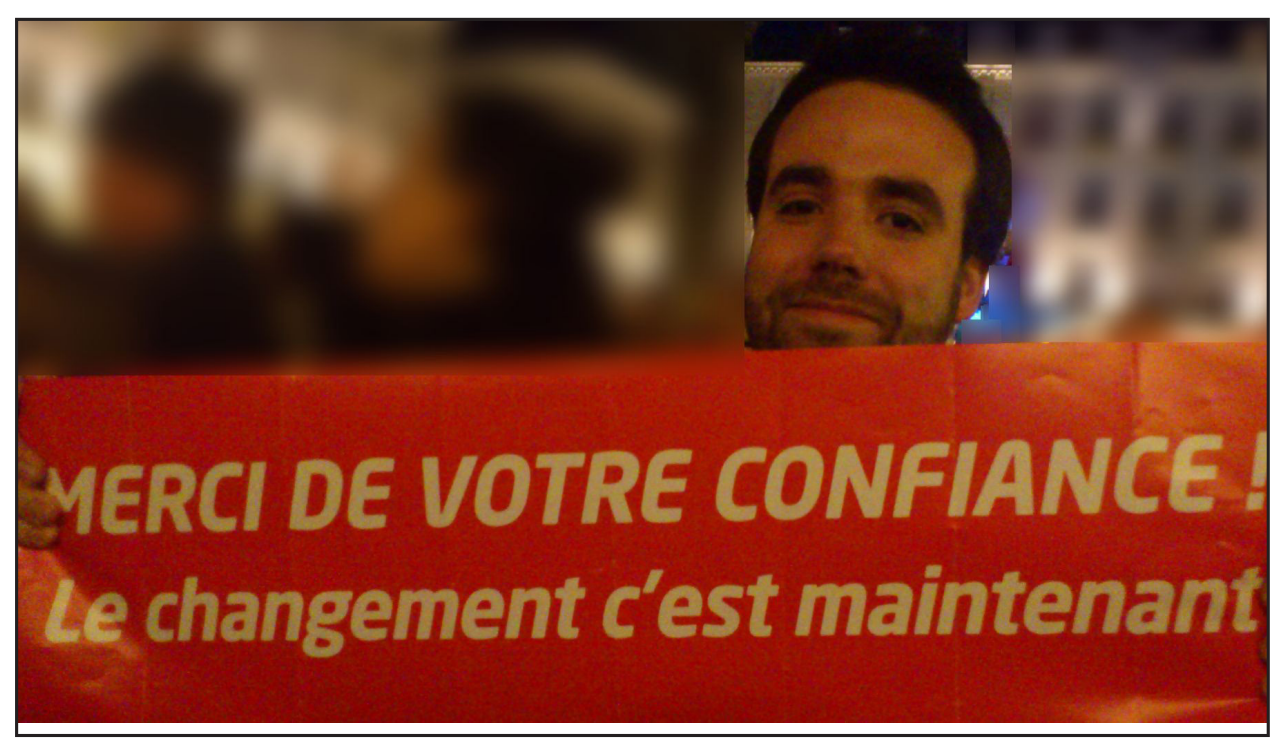

L'auteur, les traits tirés et le regard fatigué après une campagne électorale éreintante [Document personnel de l'auteur] Les campagnes représentent également une opportunité, pour les responsables du parti, de repérer les militants les plus motivés, les plus disponibles, et de sélectionner ceux susceptibles de «monter » et d'occuper des responsabilités (Bargel, 2011). Or, sans le savoir, je correspondais au profil alors recherché par un parti ayant l'ambition de « recruter » de jeunes militants. Je possédais les compétences attendues par les responsables de la Section : la «bonne volonté militante », la connaissance du « territoire », la « disponibilité », la « jeunesse ».

C'est ainsi que le Secrétaire de la Section intégrée pendant la campagne me propose, seulement quelques semaines après mon arrivée au PS, d'intégrer la Commission Administrative ${ }^{4}$ (CA) de la Section et de devenir responsable de l'organisation de la vie militante sur un secteur géographique. En l'espace de quelques mois et d'une campagne électorale, je devenais ainsi membre d'une CA à laquelle il n'est statutairement possible d'accéder que par l'élection.

\section{De l'évidence d'une adhésion}

La rapidité de mon accession à des responsabilités partisanes peut paraître, en soi, relativement étonnante. À ce stade du texte, le lecteur s'est d'ailleurs peut-être déjà inquiété intérieurement de la non-évocation de mon adhésion au PS : «Sans doute préfère-t-il la cacher afin que je ne puisse douter de son objectivité », se dit-il peut-être ? Non : à ce stade de ma « carrière » (Filleule, 2009), je n'étais pas encore adhérent ; mais le plus déconcertant sans doute, c'est que personne ne semblait le savoir!

Un « activisme » aussi important que le mien, en effet, ne pouvait être celui d'un « simple » sympathisant. Au cours de la campagne électorale précédemment mentionnée, Jean-Paul, Secrétaire de Section, même lorsque j'explique clairement que je ne suis pas adhérent, semble ne pouvoir entendre la précision :

Lors du collage, Jean-Paul me demande : «Donc toi t’as adhéré là, juste... » Moi : «J'ai pas encore adhéré. » [Quelques secondes de silence révélatrices de sa surprise.] «Et nos histoires de Congrès, de courants, ça t’a pas rebuté pour adhérer?»

Moi : « Pour l'instant non (Rire).»

[Journal de terrain - 13 mars 2010]

Si j'étais devenu militant, je n'étais donc pas encore devenu adhérent. Devenir militant (ou du moins « jouer » au militant) pour les besoins d'une enquête ethnographique me semblait acceptable ; il en était autrement de l'adhésion qui me paraissait contrevenir à la « neutralité axiologique » exigée de tout bon chercheur.
4 Instance de direction de la Section. 
Lors des semaines suivantes, je me comporte toutefois comme un adhérent exemplaire, faisant par exemple preuve d'une assiduité sans faille aux Assemblées Générales (AG). En revanche, n'étant pas adhérent, il ne m'est pas possible de prendre part aux votes internes; ce qui ne surprend pas mes enquêtés qui mettent cette attitude sur le compte du caractère récent d'une adhésion qui leur semble évidente ${ }^{5}$. Rendue possible par le flou régnant au PS sur les différents niveaux de militantisme - adhérents, militants, sympathisants - qui y coexistent (Lefebvre, 2013), cette situation me rend toutefois mal à l'aise à l'égard d'« enquêtés » devenus des «camarades ». Le « tourment » que je ressens alors quant à la position occupée sur le « terrain » se résume à une question : est-il nécessaire que je prenne ma carte pour pouvoir continuer à mener mon enquête sans avoir le sentiment de trahir mes « enquêtés » ?

L'accès à des responsabilités partisanes allait cependant m'obliger à mettre rapidement un terme au dilemme qui était le mien. Un mois après être devenu membre de la CA, je suis en effet contraint, lors d'une soirée de vote interne, d'avouer à Jean-Paul que je n'ai toujours pas adhéré ; ce dernier, estomaqué, me fait savoir que je suis dans l'obligation d'adhérer pour intégrer les instances du parti.

Lorsque j'arrive au bureau de vote, Antoine, un militant avec lequel j'ai sympathisé, me salue et m'invite à voter. Je lui réponds, comme lors de chaque vote, que «je ne peux toujours pas voter ». Il semble étonné et je lui explique que «je ne suis toujours pas adhérent ».

Jean-Paul est à côté et tombe des nues : « T'es toujours pas adhérent ?! » Après quelques secondes de silence, il me dit : « Il faut qu'on te donne un coup de main, il faut régler ça. » Je lui demande « les papiers »; il me répond : « Les papiers, ça va te coûter de l'argent, on va le faire sur internet ». Romain arrive et demande ce qu'il se passe. Jean-Paul lui répond : «Rien, c'est entre nous ». Il n'en revient toujours pas : «T'as pas encore déposé d'adhésion? Si tu le fais par internet, ça te coûte vingt euros. En déposant les papiers, le plus bas, c'est vingt-quatre euros. Donc t'as intérêt à le faire par internet. » Romain prend finalement part à la discussion ; il est très étonné. Antoine également. [...] Un peu plus tard, Jean-Paul revient me voir pour régler les modalités de mon adhésion ; il est inquiet : « Il ne faut quand même pas tarder. Parce que sinon, je vais avoir des problèmes en CA. Je ne peux pas te faire rentrer si tu n'es pas adhérent. » Jean-Paul me demande ensuite si je serai présent le lendemain pour le deuxième tour probable pour la désignation de la tête de liste pour les élections sénatoriales. Je lui réponds que je n'avais pas prévu de venir mais que je passerai peut-être en fin de soirée. Il me redemande alors : « Tu votes? Demain?»

Je me vois dans l'obligation de lui rappeler que je ne peux pas voter ; mais il n'en revient toujours pas : « Ah bah oui... J'arrive pas à m'y faire. »

[Journal de terrain - 2 décembre 2010]

Je n'avais donc plus le choix : pour continuer à mener mon immersion, je me devais d'adhérer ; ce que je fis dès le lendemain. À l'image de Daniel Bizeul lors de son immersion au Front National (Bizeul, 2003), il me fallait à nouveau donner des gages de loyauté envers des enquêtés qui me faisaient à présent confiance. L'adhésion était devenue inévitable pour la poursuite de l'enquête et pour me maintenir sur un terrain de recherche que j'allais - je l'ignorais alors - être amené à fréquenter six années durant.
5 Une ancienneté de six mois est exigée pour prendre part aux scrutins internes. 


\section{Négocier le maintien sur le terrain : quand l'immersion mène à l' « enclicage »}

L'« enclicage »-l'insertion du chercheur « dans certains réseaux et pas dans d'autres » conduisant celui-ci à « être assimilé, souvent malgré lui, mais parfois avec sa complicité, à une "clique" ou une "faction" locale » (Olivier de Sardan, 1995, p. 16) - est souvent présenté comme un «biais » de l'enquête ethnographique, si ce n'est comme un «problème » ou un « risque ». Il est pourtant souvent difficilement contournable, notamment dans le cas d'une immersion ethnographique de longue durée en milieu partisan (Bué, 2010). L'« enclicage » est ainsi devenu progressivement inéluctable dans la négociation implicite menée avec mes « enquêtés » pour me maintenir sur le terrain.

\section{Négocier sa place dans les « jeux internes »}

« Se situer » et « être situé » dans un courant constitue l'étape suivante du processus d'insertion progressive à la vie partisane. Façon de jauger les nouveaux entrants, et potentiels futurs concurrents dans les luttes internes, les incitations à se positionner se font de plus en plus pressantes lorsque l'on évolue dans la hiérarchie.

Au départ, c'est pourtant mon absence dans les luttes entre courants qui incite les dirigeants de la Section à me confier des responsabilités. Assidu lors des AG, je suis identifié comme un militant motivé et susceptible de progresser. Mais n'y prenant jamais la parole, je ne peux être identifié comme étant proche de tel courant plutôt que de tel autre. Je corresponds parfaitement au profil recherché pour renouveler les instances de la Section : « des gens motivés, impliqués, qui ne sont pas trop dans les luttes de courant $»^{6}$.

L'accès aux instances de militants « non situés » pouvant cependant susciter quelques réticences, je comprends rapidement qu'il me faudra prendre position. Je ressens par ailleurs, chez les responsables des différents courants, une volonté de m'enrôler :

Au cours de la première réunion de la CA à laquelle j'assiste, un militant interroge Jean-Paul sur les nouveaux adhérents invités à l'intégrer : représentent-ils une Motion ? Il répond par la négative en précisant que là n'est pas le sujet. Un brouhaha s'ensuit. Françoise, élue membre du courant «Un Monde d'Avance » (UMA) représentant l'aile gauche du parti, rappelle l'importance que constitue à ses yeux la désignation des membres des instances du parti en proportion des résultats obtenus par les différentes Motions lors des Congrès ${ }^{7}$. Jean-Paul rappelle que, même si son rôle est d'être vigilant quant aux équilibres politiques, il lui est impossible de me demander ma position deux ans après le Congrès de Reims de 2008. Il termine, cependant, en soulignant : « Il a peut-être les idées très claires... ».

L'élue de l'aile gauche rebondit, en riant: « Ce n'est quand même pas quelqu'un de complètement...».

Jean-Paul rit à son tour : «Je vais faire sa formation politique ${ }^{8}$.

Tout au long de la conversation, peu à l'aise et ne sachant pas réellement quelle attitude adopter, je ne réponds pas et laisse (involontairement) planer le doute quant à mon positionnement.

[Journal de terrain - Novembre 2010]

Progressivement, par la participation aux AG notamment, j'apprends à « situer » les adhérents en fonction des courants ; mais aussi à me positionner dans les « jeux » internes. Lors des premières réunions auxquelles j'assiste, je suis rapidement séduit par les interventions de Jean, responsable départemental du courant «UMA », son discours faisant écho à des valeurs
6 C'est ainsi que Jean-Paul me dresse, lors du rendez-vous où il me propose d'intégrer la CA, le « portrait-robot» des militants recherchés pour renouveler la Section.

7 Les Congrès visent à définir l'orientation politique du PS. La répartition des postes dans ses instances nationales et locales s'effectue ensuite proportionnellement aux résultats obtenus par les Motions, textes d'orientation portés par les différents courants.

8 L'élue de l'aile gauche sousentend ici que mes « antécédents familiaux » lui permettent de supposer de moi une certaine proximité avec le courant auquel elle appartient (rappelons que mon père a été, pendant plusieurs années, adhérent du PS et militant de son aile gauche). Le Secrétaire de Section laisse entendre que rien n'est joué et qu'il espère me faire adhérer au courant majoritaire qu'il représente. 
intériorisées au cours de ma socialisation, primaire (familiale) et secondaire (étudiante $^{9}$ ), à la politique. Je suis cependant réticent, dans cette phase d'accès à des responsabilités et de découverte des enjeux partisans, à exprimer une quelconque proximité avec un courant.

Ce n'est qu'en participant très activement, début 2011, à la campagne cantonale de Françoise que je suis finalement étiqueté comme membre de ce courant. Sans que j'en ai pleinement conscience, le simple fait de participer à cette campagne pouvait être interprété comme un probable indice de ralliement à l'aile gauche, aussi bien par ses membres que par les tenants du courant « majoritaire».

Une fois «situé » comme membre de cette sensibilité, il me fallait confirmer ma loyauté à l'égard de ses militants et responsables, ce que j'ai l'occasion de faire lors de la campagne des Primaires, en septembre et octobre 2011. Les responsables de la campagne de M. Aubry - candidate soutenue notamment par «UMA »- me confient des tâches militantes ( « espionner » une réunion des partisans de F. Hollande, par exemple) me permettant de faire mes preuves de loyauté.

C'est donc à cette occasion que je suis définitivement identifié comme appartenant à l'aile gauche ; ce qu'illustre l'extrait suivant de mon journal de terrain :

Ils me font confiance [...]. Mes « camarades » peuvent maintenant me situer, m'attribuer une étiquette : je suis « aubryste», appartenant à l'aile gauche du PS (comme me l'a fait plusieurs fois remarquer Antoine, militant du courant majoritaire).

[Journal de terrain - Septembre 2011]

\section{Un militant-chercheur happé par son terrain : comment devient-on collaborateur d'élus?}

Un investissement aussi important que le mien dans le militantisme ne pouvait passer inaperçu, y compris auprès des militants les moins impliqués. Il pouvait même parfois être interprété comme une volonté de gravir les échelons.

Au-delà des rétributions symboliques du militantisme (Gaxie, 1977), existent en effet des rétributions d'une toute autre nature auxquelles certains militants peuvent prétendre. L'obtention d'un poste de collaborateur d'élus dans une collectivité peut par exemple constituer un avenir envisageable, si ce n'est souhaité, par de jeunes militants en fin d'études envahis par le doute quant à leur avenir professionnel. L'évolution « militant - collaborateur d'élus - élu » constitue un processus considéré comme «normal », « classique», ou du moins imaginable pour de jeunes militants.

Dans un bar où nous partageons un verre après le collage, Clément, jeune militant de l'aile gauche du parti, me confie qu'il ne souhaite pas poursuivre ses études universitaires de droit. Il en vient à évoquer le travail de collaborateur d'élus : « Avec un Master de sociologie, tu peux être collaborateur d'élus ». Il fait référence à Olivier, l'un de mes anciens étudiants, titulaire d'une Maîtrise en droit et d'une Licence de sociologie, et alors collaborateur d'élus au Groupe socialiste [d'une collectivité locale].

Pour Clément, un tel poste ne peut cependant être que «provisoire » et ne peut être envisagé comme un avenir professionnel stable et durable : «C'est un poste que tu peux garder six ans et puis... ». Antoine termine la phrase de Clément : « Tu ne peux pas le garder quand toi, tu es élu. »

[Journal de terrain - Septembre 2011]

Ce n'est cependant alors pas mon cas : à ce stade de l'immersion, je n'envisage aucunement cette «carrière ». Bien que la dimension calculatrice du militantisme existe chez un certain nombre de (jeunes) militants, c'est
9 À l'Université, je participe, de manière intensive mais passive, à l'ensemble des mouvements étudiants (contre le CPE et la LRU). Je n'ai pour autant jamais adhéré à une organisation politique de jeunesse ou à un syndicat étudiant. 
souvent dans le cours même de l'engagement que sont découvertes des possibilités non anticipées d'accéder à des rétributions symboliques et matérielles (Bargel, 2011 ; Bargel, 2014).

C'est ainsi pendant la campagne des Primaires que la proposition m'est faite, une première fois, de devenir collaborateur d'élus au Groupe socialiste d'une collectivité locale. Rappelons que je suis alors identifié comme membre de l'aile gauche ; ce n'est donc pas un hasard si c'est Jean, son responsable départemental, élu dans cette collectivité, qui me propose le poste :

Après une action de la campagne de $\mathrm{M}$. Aubry, Jean me fait une proposition à laquelle je ne m'attendais pas et qui me met quelque peu dans l'embarras... : «Je ne sais pas quels sont tes projets professionnels, mais est-ce qu'un boulot au Groupe socialiste [dans telle collectivité], t'intéresserait? ». Il précise : «C'est un boulot à plein temps ».

Surpris, je réponds que j'ai « un poste à la fac ». Il semble à son tour étonné : il l'ignorait. «Il y a un poste qui se libère, donc je pensais à toi, qui pouvait avoir les compétences politiques, et qui sait sans doute écrire », m'explique-t-il. Il poursuit : « Bon, écoute, non. Donc t'as déjà un poste, je ne savais pas. » [Journal de terrain - Octobre 2011]

Occupant un poste d'ATER à mi-temps, il m'est alors impossible d'accepter ce qui m'est présenté comme un «boulot à temps plein ». La proposition ne me laisse cependant pas insensible et je laisse entendre que si l'occasion devait se présenter à nouveau, je pourrais être susceptible de l'accepter. J'y voyais notamment la possibilité de poursuivre ma thèse dans de bonnes conditions financières en travaillant dans une collectivité pouvant par ailleurs constituer un poste d'observation intéressant.

Ce n'était donc que partie remise : en septembre 2012, un autre poste se libère, il m'est de nouveau proposé et je suis recruté comme collaborateur d'élus, un poste que je vais occuper pendant trois ans (de 2012 à 2015).

\section{Négocier (avec soi-même) la sortie du terrain : la résolution du « tourment militant »}

À ce moment-là de la recherche, ce rebondissement a toutes les chances de se muer en un « raté » de l'enquête. Devenu militant « professionnalisé », je me retrouve dès lors entièrement happé par le terrain. En me contraignant à mettre un temps ma thèse « entre parenthèses $»^{10} \mathrm{en}$ raison de l'intensification du militantisme qui accompagne nécessairement toute forme de professionnalisation politique (Lefebvre, Sawicki, 2006), cet « incident » met en danger la poursuite même d'une recherche dont d'objet devient de plus en plus vague.

\section{Le « tourment » ou l'usure du militant}

Si le statut de salarié rend possible un accroissement de l'engagement militant, il peut cependant être également source de tourment. Le collaborateur d'élus est souvent partagé entre, d'un côté, la dépendance envers des élus ou des responsables partisans grâce auxquels il peut « vivre de » la politique et, de l'autre, la fidélité qu'il souhaite conserver envers des valeurs et des convictions qui lui permettent de «vivre pour » la politique. La tension inhérente au statut de collaborateur d'élus (Delasalle, 2019) peut ainsi conduire le militant à ressentir une certaine « usure » de son militantisme.

Dans mon cas, cette « usure » s'est accompagnée d'un autre « tourment » : celui du chercheur pressé par l'institution d'achever une thèse débutée quelques années plus tôt. Il me fallait effectuer un choix entre la poursuite d'une « carrière » politique et l'achèvement de ma recherche. Cette « double-tension »
10 Comme je le répondais aux rares enquêtés se souvenant de cette part de mon identité que je tentais de faire oublier et sur laquelle ils m'interrogeaient parfois. 
m'a finalement conduit à un « désengagement militant» (Fillieule, 2005) progressif mettant fin à une « carrière » politique ascendante jusqu'alors marquée par l'accès à des responsabilités partisanes toujours plus importantes ${ }^{11}$. À l'occasion du Congrès de Poitiers (juin 2015), dans un contexte national marqué par les tensions croissantes entre les « frondeurs » et les soutiens inconditionnels du Président de la République, je préfère ainsi renoncer à briguer le poste de Secrétaire de Section auquel les militants de l'aile gauche du parti me pressaient pourtant de candidater. À l'approche d'élections locales dont l'issue incertaine me laisse dans le doute quant à mon avenir professionnel, craignant que mon affiliation aux « frondeurs » puisse porter « préjudice » à mon avenir professionnel, je ne souhaite pas risquer, en montant en première ligne dans une « lutte interne » qui s'annonce « violente», de voir des portes se fermer devant moi ${ }^{12}$.
11 En 2013, suite à la démission de Jean-Paul, j'accède au poste de Co-Secrétaire de Section par intérim. En 2015, je deviens directeur de campagne de candidats aux élections départementales. Au cours de cette période, je suis également, au nom de l'aile gauche du parti, membre suppléant du Conseil Fédéral (CF), instance départementale de direction et de décision.

12 Une élue de la motion majoritaire, de qui j'étais proche, m'a confirmé après le Congrès : « Oui, on t'aurait flingué. C'est la politique...».

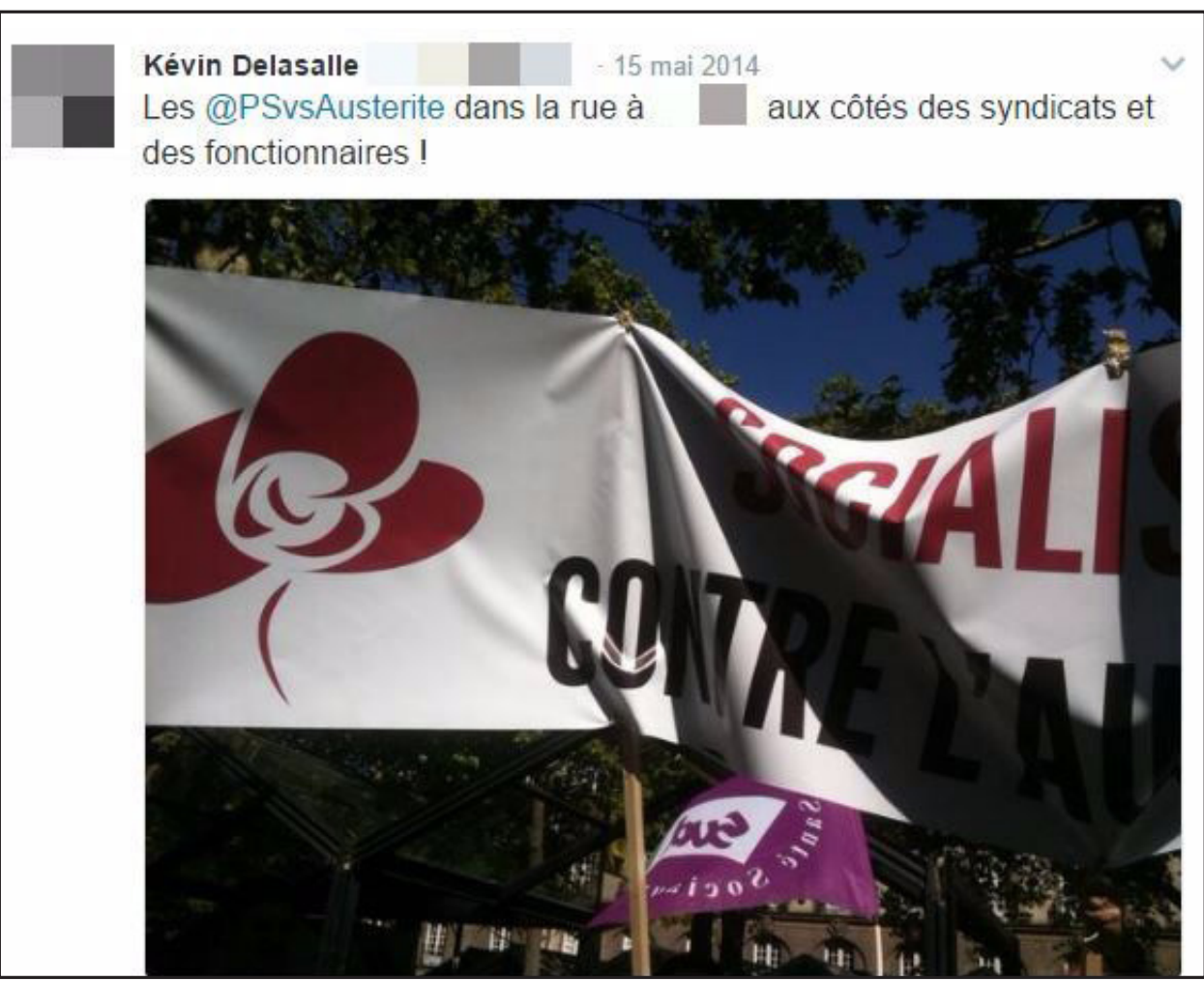

2014-2015 : un « tourment militant » vécu intimement Mai 2014, une dizaine de « Socialistes contre l'austérité » défilent derrière une banderole [Tweet personnel de l'auteur] 


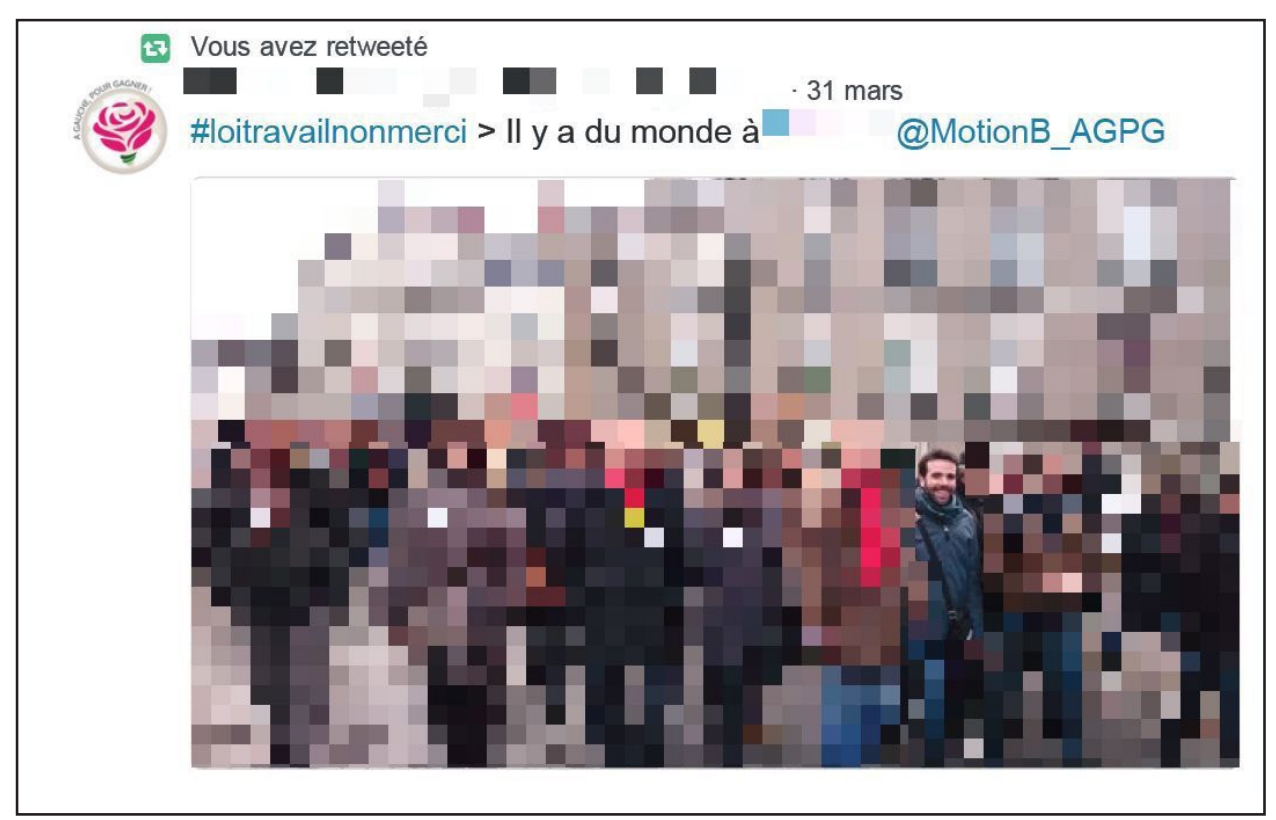

Mars 2015, participation de militants socialistes (dont l'auteur) à une manifestation contre la « Loi Travail » [Tweet publié sur un compte militant public]

Quelques mois plus tard, las de ces tensions internes, je prends vivement à partie, au cours d'une $\mathrm{AG}$ de Section rassemblant une petite trentaine de militants, un Député partisan de la « Loi Travail ». En représailles, ce Député m'adresse une « lettre ouverte », envoyée aux 300 adhérents de la Section. Celle-ci ne fait qu'amplifier un processus de « désengagement militant » dans lequel je me trouvais pris depuis plusieurs mois. Durant quelques semaines, je n'assiste ensuite qu'avec intermittence aux réunions de « courant », tout en évitant soigneusement les AG et Conseils Fédéraux, avant de finalement cesser toute activité militante pour me consacrer pleinement à l'écriture de ma thèse.

Ce processus de « désengagement militant » progressif m'a finalement permis de me (re)mobiliser en tant que chercheur. Si, en effet, «l'impossibilité de reconvertir ailleurs son militantisme et l'inexistence de possibles latéraux » (Lefebvre, $2008: 236$ ) permettent souvent d'expliquer le maintien, malgré les « dissonances cognitives », dans un engagement duquel il serait « coûteux » de sortir, c'est au contraire l'existence d'un « possible latéral » qui rend possible, à ce moment-là de ma « carrière » militante, mon « désengagement » : avec l'écriture de ma thèse, je suis en effet à nouveau « engagé » dans une autre « sphère de vie » (Passy, 2005), le milieu universitaire.

\section{Se faire l'ethnographe « indigène » du « tourment militant»}

La progression de l'immersion vers un militantisme professionnalisé n'était pas envisagée lorsque la recherche a débuté ; elle a cependant joué un rôle déterminant par la suite. Cette évolution m'a effectivement conduit à réorienter mon travail doctoral vers un objet de recherche peu exploré par la sociologie du militantisme : l'engagement politique dans sa dimension malheureuse.

Ma trajectoire singulière rendait possible une sociologie «par le soi» de ce qu'il convient de nommer le «tourment militant». Les rebondissements connus par ma recherche m'ont permis d'explorer des données auxquelles seul un tel positionnement pouvait me permettre d'accéder. En prenant activement part aux «jeux » partisans, en découvrant les échanges de « coups » et les coûts du militantisme, en étant profondément taraudé par ma propre expérience professionnelle et militante, j’ai intimement expérimenté la dimension mal- 
heureuse du militantisme. Cette expérience personnelle m'a offert un accès à cette intimité difficilement explorable par d'autres voies méthodologiques que celle de l'immersion ethnographique.

Début juin 2016, alors que le mouvement contre la « Loi Travail » bat son plein : Fête de Section. Ambiance morose. Le cœur n'est à la fête pour personne. [...] Pendant le repas, l'ambiance est pour le moins tendue. Avec les camarades de la Motion, nous avions réservé la quasi-totalité d'une table, à part des « autres ». [...]

Après le repas, le beau-fils de Carine s'occupe de la musique. Le premier morceau, «IWill Survive », fait se lever et danser quelques militants; mais pas notre table. Jean, pourtant, aime chanter et danser ; il est habituellement capable de se lâcher sur cette chanson, dit-il. Pas ce soir. Carine demande ensuite à son beau-fils de diffuser des chants révolutionnaires. «L'Internationale » : plusieurs militants viennent chercher Jean, lui demandant de venir chanter. Mais il ne veut pas faire la fête avec « les autres ». Impossible pour lui, dans le contexte actuel, de faire comme si de rien n'était. Malgré tout, les militants de la table chantent et lèvent le poing ; tout en restant assis. Françoise adapte les paroles à des slogans contre la « Loi Travail ». [...] Jean, le poing levé, regarde un Député dans le blanc des yeux. Celui-ci ne peut que les baisser dans son assiette de taboulé. [...]

Aux alentours de 22h30, Jean souhaite que nous partions. Il nous invite à aller chez eux, avec « les nôtres ». [...] Nous sommes quelques-uns à nous retrouver chez Françoise et Jean (Charlotte, Emmanuel, Lucie, Carine...). À l'issue de cette soirée morose, nous avions besoin de nous retrouver « entre nous ». [...] Jean a envie de chanter : il sort tous ses CD sur la table du salon, pendant que Françoise prépare pour tout le monde des mojitos avec la menthe du jardin. Tous les classiques y passent : les chants révolutionnaires, « Bella Ciao », la Compagnie Jolie Môme, Reggiani, Joan Baez, Bob Dylan, les Beatles... [...].Jean fait son « vieux con », comme il dit, nous racontant les histoires des chansons qu'il nous diffuse et les souvenirs personnels qui y sont parfois rattachés. Son premier disque marquant un signe de rupture et de rébellion par rapport à son éducation catholique, c'est « Aufray chante Dylan ». Il a toujours le vinyle et le sort fièrement pour nous en faire écouter quelques titres. [...]

Jean, ce soir, nous a ainsi fait partager ses «moments de nostalgie ». Les yeux parfois un peu embués, Jean et Françoise ont chanté, nous ont fait part d'anecdotes et d'histoires liées à leur passé militant commun. Comme si, dans cette période tourmentée du socialisme, ils avaient besoin - et nous en avions tous besoin... - de se rappeler pourquoi ils se battent, de se souvenir pourquoi ils militent, de redonner un sens à leur engagement.

Avant de partir, nous nous sommes tous mis debout, nous sommes tenus par les épaules, et avons fredonné, a capella (certains, comme moi, avec les paroles sous les yeux), le « Chant des Partisans ».

[Journal de terrain - Juin 2016]

Construit progressivement par mon expérience ethnographique et participante du terrain socialiste, par le trouble lié, d'une part, à l'évolution du PS et du contexte politique au cours de mon enquête et, d'autre part, à mon positionnement particulier de militant-ethnographe, l'objet final de la thèse est ainsi intimement lié à ma trajectoire d'engagement partisan personnel.

Le malaise ressenti intérieurement à son issue ne pouvait trouver sa résolution que dans une prise de distance avec un engagement dans lequel j'ai pourtant un temps eu la possibilité (et la tentation) de me professionnaliser durablement (Sorignet, 2011 ; Wacquant, 2011). Afin de négocier avec moimême, à présent ancien militant, la sortie de mon terrain, il me fallait (re) devenir chercheur et me faire l'ethnographe « indigène » du milieu auquel j'ai fini par appartenir (Bensa, 2008). 


\section{Conclusion : l'ethnographie des « carrières » de militants tourmentés}

La notion de « carrière » (Hugues, 1996 ; Becker, 1985) est particulièrement féconde pour analyser un « désengagement » militant qu'Olivier Fillieule propose de saisir de manière « processuelle » afin « de travailler ensemble les questions des prédispositions au militantisme, du passage à l'acte, des formes différenciées et variables dans le temps prises par l'engagement, de la multiplicité des engagements le long du cycle de vie (défection(s) et déplacement(s) d'un collectif à l'autre, d'un type de militantisme à l'autre) et de la rétractation ou extension des engagements » (Fillieule, 2001, p. 201). L'approche théorique et méthodologique proposée par Olivier Fillieule s'est avéré heuristique pour analyser ma propre trajectoire - ainsi que je l'ai esquissé dans le présent article -, comme celles d'autres militants à l'aune de leurs émotions (Bachelot, 2015) et des expressions différenciées et évolutives de leur(s) « tourment(s)».

Le processus de « désengagement » se déroule généralement sur le temps long (Willemez, 2004) et, comme le note Catherine Leclercq, condamne souvent « le chercheur à travailler sur du matériau reconstruit » et à mener une « analyse rétrospective ». La raison en est la difficulté à « mettre en œuvre une enquête longitudinale » qui, seule, permettrait de « saisir véritablement des défections "en train de se faire" ». La seule façon de mener à bien l'observation de processus de « désengagement» serait en effet « d'étudier un collectif sur la longue durée en espérant "voir partir" un certain nombre de ses membres » (Leclercq, 2008, p. 7).

Est-il besoin de préciser que tel n'était pas mon secret espoir au début de l'immersion ethnographique qui a été la mienne et dont les grandes lignes ont été retracées? Le contexte national d'un PS en crise s'est chargé de faire émerger le tourment des militants socialistes et de me le faire ressentir intimement.

La recherche que j'ai menée m'a toutefois permis de me départir des biais immanquablement induits par une enquête rétrospective et menée exclusivement par entretiens (Fillieule, Broqua, 2005 ; Sommier, 2005). C'est bel et bien l'immersion ethnographique, totale et de longue durée, qui m'a, au final et à mon corps défendant, permis de saisir, au travers de « carrières militantes » observées sur le temps long, le processus menant progressivement au ressenti du « tourment » puis à son expression éventuelle, ou au contraire à son enfouissement. 


\section{Bibliographie}

AÏT-Aoudia Myriam, BARgEL Lucie, ETHuin Nathalie, MAssicARd Élise et Petitfils Anne-Sophie, 2010, «Franchir les seuils des partis. Accès au terrain et dynamique d'enquête », Revue Internationale de Politique Comparée, $\mathrm{n}^{\circ} 17-4$, p. 15-30.

BACHELOT Carole, 2015, « Les émotions au sommet des partis : le cas du Parti Socialiste », Communication au Congrès de l'Association Française de Sociologie, Aix-en-Provence.

BACHELOT Carole, 2011, «L'ethnographie des dirigeants de partis : le cas du PS », Genèses, nº 83, p. 118-132.

BAJARD Flora, 2013, « Enquêter en milieu familier. Comment jouer du rapport de filiation avec le terrain ?», Genèses, $n^{\circ}$ 90-1, p. 7-24.

BARgel Lucie, 2014, «Apprendre un métier qui ne s'apprend pas. Carrières dans les organisations de jeunesse des partis », Sociologie, $\mathrm{n}^{\circ} 5-2$, p. 171-187.

BARGEL Lucie, 2011, « S'attacher à la politique. Carrières de jeunes socialistes professionnels », Sociétés Contemporaines, $\mathrm{n}^{\circ}$ 84, p. 79-102.

BECKer Howard, 1985 (1963) Outsiders. Études de sociologie de la déviance, Paris, Éditions Métailié, 248 p.

Bensa Alban, 2008a, « Père de Pwädé. Retour sur une ethnologie au long cours », dans Fassin Didier et BENSA Alban (dir.), Les politiques de l'enquête. Épreuves ethnographiques, Paris, La Découverte, p. 19-39.

BENSA Alban, 2008b « Conclusion. Remarques sur les politiques de l'intersubjectivité », dans Fassin Didier et Bensa Alban (dir.), Les politiques de l'enquête. Épreuves ethnographiques, Paris, La Découverte, p. 323-328.

Bizeul Daniel, 2003, Avec ceux du FN. Un sociologue au Front National, Paris, La Découverte, 300 p.

Bizeul Daniel, 1998, « Le récit des conditions d'enquête : exploiter l'information en connaissance de cause », Revue Française de Sociologie, $\mathrm{n}^{\circ}$ 39-4, p. 751-787.

BRoQUA Christophe, 2009a, « L'ethnographie comme engagement : enquêter en terrain militant », Genèses, $n^{\circ}$ 75, p. 109-124.

BRoQua Christophe, 2009b, « Observation ethnographique », dans Fillieule Olivier, Mathieu Lilian et Péchu Cécile (dir.), Dictionnaire des mouvements sociaux, Paris, Presses de Sciences Po, p. 379-386.

BuÉ Nicolas, 2010, «Gérer les relations d'enquête en terrains imbriqués. Risque d'enclicage et distances aux enquêtés dans une recherche sur une coalition partisane locale », Revue Internationale de Politique Comparée, $\mathrm{n}^{0}$ 17-4, p. 77-91.

CARATini Sophie, 2017, «Conclusion. Réflexion comparative sur quelques postures anthropologiques vécues de l'ailleurs et du proche », Émulations, $\mathrm{n}^{\circ} 22$, p. 127-134.

DeLASAlle Kévin, 2019, « Loyauté ou exit de collaborateurs d'élus français. L'influence de la (dés)obéissance sur leurs trajectoires professionnelles », Émulations, en ligne, DOI : 10.14428/emulations. varia. 020

Delasalle Kévin, 2017, Le tourment militant. Ethnographie de l'engagement partisan au Parti Socialiste (2010-2016), Thèse de Doctorat en Sociologie, Université de Nantes.

FASsIN Didier, 2008, «Introduction. L'inquiétude ethnographique », dans FASsIn Didier et Bensa Alban, Les politiques de l'enquête. Épreuves ethnographiques, Paris, La Découverte, p. 6-15. 
FAVRET-SAADA Jeanne, 1977, Les mots, la mort, les sorts, Paris, Gallimard, $332 \mathrm{p}$.

Fillieule Olivier, 2009 « Carrière militante », dans Fillieule Olivier, Mathieu Lilian et PÉchu Cécile (dir.), Dictionnaire des mouvements sociaux, Paris, Presses de Sciences Po, p. 85-94.

Fillieule Olivier (dir.), 2005, Le désengagement militant, Paris, Belin, 319 p.

FILlieule Olivier, 2001, «Propositions pour une analyse processuelle de l'engagement individuel », Revue Française de Science Politique, $\mathrm{n}^{\circ} 51$, p. 199-215.

FILlieule Olivier et BroQua Christophe, 2005, « La défection dans deux associations de lutte contre le sida : Act Up et AIDES », dans Fillieule Olivier (dir.), Le désengagement militant, Paris, Belin, p. 189-228.

GAXIE Daniel, 1977, «Économie des partis et rétributions du militantisme », Revue Française de Science Politique, n ${ }^{\circ}$ 27, p. 123-154.

Hugues Everett C., 1996, Le regard sociologique, Paris, Éditions de l'EHESS, $345 \mathrm{p}$.

LECLERCQ Catherine, 2008, Histoires d'« ex». Une approche sociobiographique du désengagement des militants du Parti Communiste Français, Thèse de Doctorat de Sciences Politiques, Institut d'Études Politiques de Paris, 1018 p.

LEFEBVRe Rémi, 2013, « Le militantisme socialiste n'est plus ce qu'il n'a jamais été. Modèle de "l'engagement distancié" et transformations du militantisme au Parti Socialistes », Politix, $\mathrm{n}^{\circ}$ 102, p. 7-33.

LEFEbVre Rémi, 2010a, «"Politiste et socialiste". Une politique d'enquête au PS », Revue Internationale de Politique Comparée, n ${ }^{\circ}$ 17-4, p. 127-139.

LEFEBVRe Rémi, 2010b, «"Petits arrangements avec son militantisme". Le désarroi identitaire des militants du Parti Socialiste », dans SuRdEz Muriel, VoegtuI Michaël et Voutat Bernard (dir.), Identifier S'identifier, Genève, Antipode, p. 225-243.

LEFebVRe Rémi, 2008, «"Militer au Parti Socialiste pour le transformer. L'engagement à la "gauche" du PS », dans GEAY Bertrand et WiLlemEZ Laurent (coord.), Pour une gauche de gauche, Bellecombe-en-Bauges, Éditions du Croquant, p. 217-237.

LEFEBVRE Rémi et SAWICKI Frédéric, 2006, La société des socialistes. Le PS aujourd'hui, Bellecombe-en-Bauges, Éditions du Croquant, 255 p.

NAUdier Delphine et Simonet Maud, 2011, «Introduction », dans NAUdier Delphine et Simonet Maud (dir.), Des sociologues sans qualités?, Paris, La Découverte, p. 5-21.

Olivier de SARdan Jean-Pierre, 1995, « La politique du terrain. Sur la production de données en anthropologie », Enquête [En ligne], $\mathrm{n}^{\circ} 1$, mis en ligne le 10 juillet 2013, consulté le 3 juin 2016, URL : http:// enquete.revues.org/263; DOI : 10.4000/enquete.263

PAssy Florence, 2005, « Interactions sociales et imbrications des sphères de vie », dans Fillieule Olivier (dir.), Le désengagement militant, Paris, Belin, p. 111-130.

SAWICKI Frédéric, 1988, «Questions de recherche : pour une analyse locale des partis politiques », Politix, $\mathrm{n}^{\circ}$ 2, p. 13-28.

SAwicki Frédéric, 1997, Les réseaux du Parti socialiste, Paris, Belin.

SAWICKI Frédéric, 2000, «Les politistes et le microscope », dans BACHIR Myriam (dir.), Les méthodes au concret. Démarches, formes de l'expérience et terrains d'investigation en science politique, Paris, Presses Universitaires de France - CURAPP, p. 143-164.

SOMmier Isabelle, 2005, « Une expérience "incommunicable" ? Les ex-militants d'extrême-gauche français et italiens », dans Olivier Fillieule (dir.), Le désengagement militant, Paris, Belin, p. 171-188. 
SoRignet Pierre-Emmanuel, 2011, « Sociologue et danseur, quand la vocation se fait double », dans NAUDIER Delphine et Simonet Maud (dir.), Des sociologues sans qualités ?, Paris, La Découverte, p. 222-240.

SchwARTz Olivier, 1993, « L'empirisme irréductible », dans ANDERSON Nels, Le Hobo. Sociologie du sans-abri, Paris, Nathan, p. 265-308.

Willemez Laurent, 2004, « Perseverare diabolicum : l'engagement militant à l'épreuve du vieillissement social », Lien social et politiques, $\mathrm{n}^{\circ} 51$, p. 71-82.

WACQUANT Loïc, 2011, « La chair et le texte : l'ethnographie comme instrument de rupture et de construction », dans NAUDIER Delphine et Simonet Maud (dir.), Des sociologues sans qualités? Paris, La Découverte, p. 201-221. 